\title{
GESTATIONAL PROFILE OF PATIENTS WITH ERYTHEMATUS LUPUS CARE IN TERTIARY SERVICE IN AMAZONAS
}

Andrezza Mendes Franco ${ }^{1, \star}$, Clara Pinheiro Martins ${ }^{1}$, Igor Oliveira da Silva¹, Camylla Rita Lima do Nascimento', Vitor Matheus Orlando Sampaio ${ }^{1}$, Camile Smith de Oliveira Brito', Ingrid dos Santos Araújo', Sebastião Barreto Falcão Neto', Sandra Lúcia Euzébio Ribeiro'

1.Universidade Federal do Amazonas, Manaus (AM), Brazil.

*Corresponding author: andrezzamf2190@gmail.com

\section{BACKGROUND}

Systemic lupus erythematosus (SLE) is an immune-mediated rheumatic disease that affects multiple organs. It predominates in females in reproductive age. It is influenced by genetic, environmental, hormonal factors, lifestyle habits, among others. Systemic lupus erythematosus can trigger serious repercussions during pregnancy, including abortions, prematurity, low birth weight, malformations, among others. Therefore, this study aims to analyze the gestational profile of patients with SLE treated at a university hospital in Manaus, state of Amazonas.

\section{MATERIALS AND METHODS}

A descriptive, retrospective and cross-sectional study in which 182 medical records of female patients diagnosed with SLE, of all age groups and under regular follow-up between 2017 and 2020 at a reference rheumatology service in Amazonas, were analyzed. Information about age, number of pregnancies, abortions, live births, gestational morbidity and complications were verified, aiming to obtain a gestational profile of this group.

\section{RESULTS}

The average age found in this group was $44.2 \pm 11.7$ years. In the gestational history, an average of $2.32 \pm 2.03$ pregnancies, $0.49 \pm 0.80$ abortions and $1.83 \pm 1.69$ live births were observed. Gestational morbidity was present in $32.9 \%$ of patients. Among the morbidity modalities, $80 \%$ had 1 or more unexplained deaths of morphologically normal fetuses during/after the 10 th week; $15 \% 1$ or more premature births of a morphologically normal neonate during/before the 34th week; and $6.7 \%$ had 3 or more consecutive and unexplained abortions, before the 10th week. The gestational complications reported in the medical records were: preeclampsia, stillbirth, gestational toxoplasmosis, specific hypertensive pregnancy disease, bacterial infections in the postpartum period, neonatal SLE, ectopic pregnancy and hydatidiform mole. In addition, 24 patients with a diagnosis of antiphospholipid antibody syndrome (APS) secondary to SLE were identified, who had the worst rates of fetal loss (mean of $0.91 \pm 1.10$ abortions) and gestational morbidity (50\%).

\section{CONCLUSION}

In line with the literature, it was noted that women with SLE of reproductive age are more prone to develop gestational complications, especially early fetal losses and prematurity. These data reinforce the need for special care and attention to SLE patients who are pregnant and/or who wish to become pregnant, in order to prevent complications and preserve quality of life. Systemic lupus erythematosus is a chronic and multisystemic inflammatory disease characterized by the presence of several autoantibodies. It is a disease that predominates in females and African Americans. Clinical manifestations can be from cutaneous and articular involvement to the occurrence of neurological events and cardiovascular graves. The aim of this study is to analyze the prevalence of SLE thromboembolic events in Amazonas. 